\title{
Evaluation of Giardia intestinalis, Entamoeba histolytica and Cryptosporidium hominis/Cryptosporidium parvum in human stool samples by the BD MAX ${ }^{\mathrm{TM}}$ Enteric Parasite Panel
}

\author{
Sadık Akgün ${ }^{1}$, Tuncay Çelik ${ }^{1}$ \\ ${ }^{1}$ Adiyaman University, Faculty of Medicine, Department of Microbiology, Adiyaman, Turkey
}

\begin{abstract}
Although the microscopic examination of stool samples remains the reference method of choice for the diagnosis of intestinal protistan infections, this method is time-consuming and requires experienced and well-trained operators. The purpose of this study was to evaluate the level of agreement between the BD MAX ${ }^{\mathrm{TM}}$ Enteric Parasite Panel (EPP) and microscopy for the detection of Giardia intestinalis (Lambl, 1859), Cryptosporidium spp. and Entamoeba histolytica Schaudinn, 1903 in stool samples. The study included faecal samples of 362 patients who were admitted to our hospital due to gastrointestinal complaints. In the microscopic examination, which was made with the native-lugol method on the stool samples that were taken from the patients, cysts, trophozoites and eggs of the parasite were examined. The diagnosis of G. intestinalis, Cryptosporidium parvum Tyzzer, 1912 and Cryptosporidium hominis Morgan-Ryan, Fall, Ward, Hijjawi, Sulaiman, Fayer, Thompson, Olson, Lal et Xiao, 2002, and E. histolytica was made in the faecal samples using the EPP assay. In the microscopic examination, Cryptosporidium spp. positive stool samples were stained with kinyoun's acid-fast. In the microscopic examination, parasites were detected in $41(11 \%)$ of the 362 stool samples. In contrast, EPP assay identified parasites in $23(6.3 \%)$ of the samples. In the microscopic examination, E. histolytica and Entamoeba dispar Brumpt, 1925 were detected in $22(6.1 \%)$ of the samples, G. intestinalis was seen in $15(4.1 \%)$, and C. parvum or C. hominis were detected in three (0.8\%); these values were five (1.4\%), 16 (4.4\%) and two (0.5\%) positive with the EPP assay. Although C. parvum or C. hominis were detected as positive in the microscopic examination of three samples, only two of the samples were positive in both EPP assay and kinyoun's acid-fast method. The EPP assay is a relatively simple test that can distinguish E. histolytica and E. dispar, but it cannot replace microscopy in the diagnosis of amoebiasis. Diagnosis for G. intestinalis and C. parvum/C. hominis with the BD MAX ${ }^{\mathrm{TM}}$ enteric parasite panel was equivalent to that with microscopy. We believe that $E$. histolytica must be diagnosed with nucleic acid amplification tests that have a high sensitivity and specificity like EPP assay in certain patient groups.
\end{abstract}

Keywords: stool microscopy, enteric parasites, Cryptosporidium spp.

According to the World Health Organisation, the second most frequent cause of morbidity and mortality in children in developing countries is diarrhea. In addition, Giardia intestinalis (Lambl, 1859), Entamoeba histolytica Schaudinn, 1903, and Cryptosporidium parvum Tyzzer, 1912/Cryptosporidium hominis Morgan-Ryan, Fall, Ward, Hijjawi, Sulaiman, Fayer, Thompson, Olson, Lal et Xiao, 2002 are the most common parasites that lead to chronic diarrhea in travellers all over the world (World Health Organization 2005, Petri et al. 2008, Pritt and Clark 2008, Checkley et al. 2015).

Light microscopy is used in the diagnosis of intestinal parasites in many laboratories. However, microscopy can lead to false conclusions, with harmless parasites being interpreted as disease-causing, or life-threatening parasites being missed (Halligan et al. 2014). Many protists are only present in small quantities in faecal samples; thus, the quality of the microscopic examination is highly dependent on the skills of the laboratory technician (Khare et al. 2014). For example, E. histolytica may appear as commensal lumen dwellers and with cysts that do not correlate with clinical symptoms. Dysentery maybe caused by bacteria or by viruses. It is therefore mandatory to consider whether any amoebas found are cysts or trophozoites and if the trophozoites are invasive and disease-causing (Ackers 2002, Shimokawa et al. 2012 ).

There are several commercially available nucleic acid amplification tests that are used in the diagnosis of the gastrointestinal pathogens (Navidad et al. 2013). Among methods developed for better diagnostics, PCR-based methods are considered the leading one, as they are highly sensitive and specific. However, these methods are technically complex and time-consuming (Tanyuksel and Petri 2003, Fotedar et al. 2007).

The BD MAX ${ }^{\mathrm{TM}}$ Enteric Parasite Panel (EPP) (Becton, Dickinson and Company, Franklin Lakes, New Jersey, 
Table 1. Microscopy and BD MAX EPP results of stool samples $(\mathrm{n}=362)$

\begin{tabular}{lcc}
\hline & Microscopy & BD MAX EPP \\
\hline Entamoeba histolytica & 22 & 5 \\
Giardia intestinalis & 15 & 16 \\
Cryptosporidium spp. $^{\mathrm{a}}$ & 3 & 2 \\
Ascaris lumbricoides & 1 & $\mathrm{NA}^{\mathrm{b}}$ \\
Negative & 321 & 339 \\
Unresolved & - & $2^{\mathrm{c}}$
\end{tabular}

${ }^{a}$ Out of 3 Cryptosporidum spp. observed under microscope 2 were observed by kinyoun's staining

${ }^{\mathrm{b}}$ Not applicable

${ }^{\mathrm{c}}$ Neither positive control nor sample gave result by microscopy

USA) is a fully automated assay providing nucleic acid extraction and simultaneous real-time amplification for the detection of E. histolytica, G. intestinalis and C. par$v u m / C$. hominis using a single platform. Use of the BD MAX Parasite Panel has recently been shown to be useful for the laboratory diagnosis of three predominant protistan parasites causing enteritis (Biswas et al. 2014, Batra et al. 2016, Madison-Antenucci et al. 2016, Parčina et al. 2018).

In the present study, the purpose was to compare the microscopic examination and EPP assay, which are used in diagnosing E. histolytica, G. intestinalis and C. par$v u m / C$.hominis, which are among enteric parasites, in patients with gastrointestinal system complaints.

\section{Samples and patient population}

In total, 362 stool samples of all patients with gastrointestinal system complaints from different clinics were admitted to the laboratory of our hospital between January 2016 and August 2018 and were retrospectively examined. Samples were collected consecutively from all patients. Of these 362 patients, 172 (48\%) were female, and 190 (53\%) were male; the average age was 43.3 years. The macroscopic examination revealed that most of the stool samples were amorphous and sloshy, and no blood or parasites were detected in any of them. The present study was conducted with the approval of the Ethics Board under Decision No. 2019/2-1 dated 20/03/2019 at Adiyaman University.

\section{Microscopy for intestinal parasites}

Microscopic examinations were performed using light microscopy on samples prepared using the wet mount method with normal saline to find motile trophozoites, cyst, ova and Lugole's iodine solution to confirm the morphology. We examined 362 stool samples using microscopy for Cryptosporidium spp. Any positive or suspected stool samples were evaluated with kinyoun's acid-fast method. All samples were kept at $-80^{\circ} \mathrm{C}$ until EPP assay was performed.

\section{Kinyoun's Acid-Fast Method}

Three stool samples contained oocysts of Cryptosporidium spp. for microscopical examination. The samples were placed in modified formalin-ethyl acetate sedimentation (the sample was placed in $10 \%$ formol and left for at least 30 minutes for fixation). The suspension was filtered through two-layer gauze into another container, and the supernatant was transferred to a centrifuge tube. Next, $3 \mathrm{ml}$ ethyl acetate was added to the suspension, and the tip of the tube was closed and shaken for 30 seconds. The suspension was centrifuged at $500 \mathrm{~g}$ for 2-3 minutes. The thin faecal smear was made and air-dried. The smear was fixed with absolute methanol for $1 \mathrm{~min}$. Then, the slide was flooded with kinyoun's carbol fuchsin stain for $5 \mathrm{~min}$. After staining, the slide was rinsed first with $50 \%$ ethanol for 3-5 s and then with distilled water. The stained smear was decolorised with $1 \%$ sulfuric acid for 2 min and was counterstained with $1 \%$ methylene blue for $1 \mathrm{~min}$. (El-Moamly and El-Sweify 2012). All samples were examined (with $1000 \times$ magnification) for the presence of oocytes for 10 minutes using immersion oil.

\section{BD MAX Enteric Parasite Panel}

The samples were processed according to the manufacturer's instructions (Becton, Dickinson and Company Franklin Lakes). Briefly, using calibrated disposable inoculating loops, 10-microlitre aliquots of vortexed stool samples were transferred into sample buffer tube. The tubes contain $1.5 \mathrm{ml}$ of an appropriate sample diluent that is formulated to minimise PCR inhibition commonly associated with stool specimens. To ensure extraction of nucleic acids from encysted protists, all specimens in SBTs were heated using a BD MAX prewarming heater. The samples were heated gradually to $37^{\circ} \mathrm{C}$ and then cooled prior to transfer to the BD Max sample racks. Following the prewarming step, manufacturer-provided unitised reagent strips, which contain all of the reagents required for nucleic acid extraction and amplification, and a microfluidics cartridge were loaded onto the BD Max instrument.

Five hundred microlitres of the diluted sample were transferred to the lysis tube of the unitised reagent strip. The sample transfer and all subsequent steps are performed automatically by the BD Max instrument. Primers included in the BD Max EPP assay detect E. histolytica, G. intestinalis and C. parvum/C. hominis. Cryptosporidium par$v u m / C$. hominis are reported as either positive or negative. Genetic targets and sequences are proprietary. Amplified products are detected with fluorophore-labeled TaqMan probes. The instrument monitors fluorescent signals at each PCR cycle and internal software provides automatic interpretation of the results. The user does not adjust the threshold for a positive result or interpret cycle threshold values.

The microscopic examination of the 362 stool samples detected $41(11 \%)$ positive samples with parasites. Out of all samples 22 were classified as E. histolytica/Entamoeba dispar Brumpt, 1925/Entamoeba moshkovskii Tshalaia, 1941 based on morphologic characteristics, $(10-15 \mu \mathrm{m}$ in diameter, spherical mature cysts tetranucleated) diagnosis upon microscopic examination, while 5 samples were found to be E. histolytica by EPP assay. Microscopic examination revealed $G$. intestinalis in $15(4 \%)$ of the samples, while $16(4 \%)$ were positive according to the EPP assay (Table 1). Although C. parvum/C. hominis were detected in 
three samples upon microscopic examination, only two of the samples were positive according to both EPP assay and kinyoun's acid-fast method. Eggs of Ascaris lumbricoides Linnaeus, 1758 were detected in one sample. According to the Kit procedure (unresolved results may be obtained in the event that specimen-associated inhibition or reagent failure prevents proper target or sample processing control amplification), two samples were evaluated as unresolved.

Entamoeba histolytica, G. intestinalis and Cryptosporidium spp. are commonly occurring parasitic protists that infect humans. In most laboratories, routine copro-parasitological examinations for helminths and protists are conducted under light microscopy using physiological saline or saline-iodine preparations. As demonstrated in other studies, molecular tests can increase the detection of pathogens while lowering both the hands-on time and the time to results (Patel et al. 2014, Buss et al. 2015). The lack of skilled clinical parasitologists and the need to perform multiple tests to adequately screen for relevant parasites only add to this challenge.

The genus Entamoeba contains many species; E. histolytica, E. dispar, Entamoeba coli, such as Entamoeba hartmanni and E. moshkovskii, which can be found in the human intestinal tract. Cysts of E. histolytica, E. dispar and E. moshkovskii are morphologically indistinguishable. Microscopy, unlike PCR, is unable to differentiate between pathogenic and non-pathogenic species of Entamoeba (see Diamond and Clark 1993, Haque et al. 2003). A significant difference was detected in our study between the microscopic examination and EPP assay results in the diagnosis of E. histolytica. This pathogenic amoeba was present in 22 of the 362 cases. However, the EPP assay identified only $5 \%$ of the samples as positive for this species. The diagnosis of E. histolytica/E. dispar/E. moshkovskii was made by considering the presence of erythrocyte phagocytosis seen upon microscopic examination. Samples that were detected as hemophagocytic forms of E. histolytica were found positive by EPP method.

There have been no prior studies conducted in our region on the use of molecular methods in the diagnosis of enteric parasites. In our previous investigation into the prevalence of intestinal parasites in primary school children, $G$. intestinalis was positive in $18 \%$ of the cases that were examined using a microscope, E. histolytica was not detected by the enzyme-linked immunosorbent assay (ELISA) method (Celik et al. 2014). In addition, G. intestinalis, which is relatively easier to diagnose using a microscopic examination, was present at a rate of $4 \%$ only. The EPP assay also found a rate of $4 \%$. Since its microscopic di- agnosis is easier compared to the other two parasites, no significant differences were detected.

Although Cryptosporidium spp. were detected in three samples upon microscopic examination, two samples were found positive by staining and EPP assay. This could be a false positivity by microscopic method or EPP assay detects only $C$. parvum and $C$. hominis, which would explain this false negative result. In contrast, this case might be another species. Since it is difficult to diagnose Cryptosporidium spp. microscopically examination, it is necessary to prepare and stain a smear to be evaluated by a specialist. In a previous study, 17 samples of 275 examined were found positive by microscopy for E. histolytica/E. dispar, and two of them were found positive by EPP and confirmed as E. histolytica. The remaining 15 negative samples by EPP were assumed to be E. dispar (Batra et al. 2016). In another study (Perry et al. 2017), it was emphasised that the specificity of $C$. parvum/C. hominis and $G$. intestinalis (99.7\% and 98.9\%, respectively) were high in EPP assay compared to the microscopic examination and EPP assay. However, it would be beneficial to compare the findings in fresh and frozen fresh stool in the diagnosis of E. histol$y$ tica. More studies are needed in this respect. In another study, the sensitivity and specificity of three commercial kits that included the EPP assay were examined. The sensitivity of $G$. intestinalis was found to be $89 \%$, which was the highest compared to the other tests. When evaluated for Cryptosporidium spp., the sensitivity was $75 \%$; however, it was $100 \%$ for the other tests. Positivity was detected in one sample in each of the three sets in the E. histolytica diagnosis (Autier et al. 2018).

In conclusion, it was determined that although there was no significant difference between the two methods for a G. intestinalis and Cryptosporidium spp. diagnosis, there was a significant difference in the E. histolytica diagnosis. Despite the fact that differentiation between E. histolytica and other species of Entamoeba cannot be made using morphology, and that a specialist cannot always make a microscopic examination, which was the case of our study, we believe that using one of the molecular methods with a high sensitivity and specificity, like EPP assay, is be better for diagnosing $E$. histolytica, especially in certain patient groups. Further studies are necessary to determine the sensitivity and specificity of the EPP assay for diagnosis E. histolytica in positive samples.

Acknowledgement. We owe thanks to Adiyaman University, Education and Research Hospital managers for their support in this study.

\section{REFERENCES}

ACKers J.P. 2002: The diagnostics implication of the separation of Entamoeba histolytica and E. dispar. J. Biosci. 27: 573-578.

Autier B., Belaz S., Razakandrainibe R., Gangneux J.P., Robert-Gangneux F. 2018: Comparison of three commercial multiplex PCR assays for the diagnosis of intestinal protozoa. Parasite. 25: 48
Batra R., Judd E., Eling J., Newsholme W., Goldenberg S.D. 2016: Molecular detection of common intestinal parasites: a performance evaluation of the BD maxtm Enteric Parasite Panel. Eur. J. Clin. Microbiol. Infect. Dis. 35: 1753-1757.

Biswas J.S., Al-Ali A., Rajput P., Smith D., Goldenberg S.D. 2014: A parallel diagnostic accuracy study of three molec- 
ular panels for the detection of bacterial gastroenteritis. Eur. J. Clin. Microbiol. Infect. Dis. 33: 2075-2081.

Buss S.N., Leber A., Chapin K., Fey P.D., Bankowski M.J, Jones M.K., Rogatcheva M., Kanack K.J., Bourzac K.M. 2015: Multicenter evaluation of the BioFire Film Array gastrointestinal panel for etiologic diagnosis of infectious gastroenteritis. J. Clin. Microbiol. 53: 915-925.

Celik T., Aydin S., Kolgelier S. 2014: Effect of intestinal parasites on weight and height percentile values in primary school students at age of 10-16 Year. Arch. Med. Invest. 12: 1-5.

Checkley W., White A.C. Jr., Jaganath D., Arrowood M.J., Chalmers R.M., Chen X.M., Fayer R., Griffiths J.K., Guerrant R.L., Hedstrom L., Huston C.D., Kotloff K.L., Kang G., Mead J.R., Miller M., Petri W.A. Jr., Priest J.W., Rooos D.S., Striepen B., Thompson R.C., Ward H.D., Van Voorhis W.A., Xiao L., Zhu G., Houpt E.R. 2015: A review of the global burden, novel diagnostics, therapeutics, and vaccine targets for Cryptosporidium. Lancet Infect. Dis. 15: 85-94.

Diamond L.S., Clark C.G. 1993: A redescription of Entamoeba histolytica Schaudinn, 1903 (Emended Walker, 1911) separating it from Entamoeba dispar Brumpt, 1925. J. Euk. Microbiol. 40: 340-344.

El-Moamly A.A., El-Sweify M.A. 2012: ImmunoCard STAT!cartridge antigen detection assay compared to microplate enzyme immunoassay and modified Kinyoun's acid-fast staining technique for detection of Cryptosporidium in fecal specimens. Parasitol. Res. 110: 1037-1041.

Fotedar R., Stark D., Beebe N., Marriott D., Ellis J., HARKNeSS J. 2007: PCR detection of Entamoeba histolytica, E. dispar and E. moshkovskii in stool samples from Sydney, Australia. J. Clin. Microbiol. 45: 1035-1037.

Halligan E., Edgeworth J., Bisnauthising K., Bible J., Cliff P., Aarons E., Klein J., Patel A., Goldenberg S. 2014: Multiplex molecular testing for management of infectious gastroenteritis in a hospital setting: a comparative diagnostic and clinical utility study. Clin. Microbiol. Infect. 20: 460-467.

Haque R., Huston C.D., Huges M., Houpt E., Petri W.A. 2003: Amebiasis. N. Engl. J. Med. 348: 1565-1573.

Khare R., Espy M.J., Cebelinski E., Boxrud D., Sloan L.M., Cunningham S.A., Pritt B.S., Patel R., Binnicker M.J. 2014: Comparative evaluation of two commercial multiplex panels for detection of gastrointestinal pathogens by use of clinical stool specimens. J. Clin. Microbiol. 52: 3667-3673.
Madison-Antenucci S., Relich R.F., Doyle L., Espina N., Fuller D., Karchmer T., Lainesse A., Mortensen J.E., Pancholi P., Veros W., Harrington S.M. 2016: Multicenter evaluation of BD max Enteric Parasite real-time PCR assay for detection of Giardia duodenalis, Cryptosporidium hominis, Cryptosporidium parvum, and Entamoeba histolytica. J. Clin. Microbiol. 54: 2681-2688.

Navidad J.F., Griswold D.J., Gradus M.S., Bhattacharyya S. 2013: Evaluation of Luminex xTAG gastrointestinal pathogen analyte-specific reagents for high-throughput, simultaneous detection of bacteria, viruses, and parasites of clinical and public health importance. J. Clin. Microbiol. 51: 3018-3024.

Parčina M., Reiter-Owona I., Mockenhaupt F.P., Vojvoda V., Gahutu J.B, Hoerauf A., Ignatius R. 2018: Highly sensitive and specific detection of Giardia duodenalis, Entamoeba histolytica, and Cryptosporidium spp. in human stool samples by the BD MAX ${ }^{\text {TM }}$ Enteric Parasite Panel. Parasitol. Res. 117: 447-451.

Patel A., Navidad J., Bhattacharyya S. 2014: Site-specific clinical evaluation of the Luminex XTAG gastrointestinal pathogen panel for detection of infectious gastroenteritis in fecal specimens. J. Clin. Mirobiol. 52: 3068-3071.

Perry M.D., Corden S.A., Lewis White P. 2017: Evaluation of the BD MAX Enteric Parasite Panel for the detection of Cryptosporidium parvum/hominis, Giardia duodenalis and Entamoeba histolytica. J. Med. Microbiol. 66: 1118-1123.

Petri W.A. Jr., Miller M., Binder H.J., Levine M.M., DilLingham R., Guerrant R.L. 2008: Enteric infections, diarrhea, and their impact on function and development. J. Clin. Invest. 118: 1277-1290.

Pritt B.S., Clark C.G. 2008: Amebiasis. Mayo Clin. Proc. 83: 1154-1159.

Shimokawa C., Kabir M., Taniuchi M., Mondal D., Kobayashi S., Ali I.K., Sobuz S.U., Senba M., Houpt E., Haque R., Petri W.A. Jr., Hamano S. 2012: Entamoeba moshkovski is associated with diarrhea in infants and causes diarrhea and colitis in mice. J. Infect. Dis. 206: 744-751.

Tanyuksel M., Petri W.A. 2003: Laboratory diagnosis of amebiasis. Clin. Microbiol. Rev.16: 713-729.

World Health Organization 2005: World health report 2005: make every mother and child count. World Health Organization, Geneva, Switzerland.

Cite this article as: Akgun S., Celik T. 2020: Evaluation of Giardia intestinalis, Entamoeba histolytica and Cryptosporidium hominis/ Cryptosporidium parvum in human stool samples by the BD MAX ${ }^{\mathrm{TM}}$ Enteric Parasite Panel. Folia Parasitol. 67: 020. 